\title{
Research on Lash-up Maintenance Technology of Engineering Equipment
}

\author{
Ming ZHANG \\ Institution of Engineering Corps of PLA, Xuzhou \\ 221004, China \\ Email: zhangming7929@sina.com \\ Chunyu DOU \\ Institution of Engineering Corps of PLA, Xuzhou \\ 221004, China
}

\author{
Zhongfeng SHI \\ Institution of Engineering Corps of PLA, Xuzhou \\ 221004, China
}

\author{
Tao FAN \\ Institution of Engineering Corps of PLA, Xuzhou \\ 221004, China
}

\begin{abstract}
The theory frame of lash-up maintenance technology of engineering equipment is brought forward from the point of view of lash-up maintenance theory, which include fault diagnosis and survey technology, damnification evaluation theory, damnification repair technology, lash-up maintenance effect evaluation theory, lash-up maintenance support informationization technology and lash-up maintenance training technology. Content and study direction of those lash-up maintenance technologies of engineering equipment are expatiated detailedly.
\end{abstract}

Keywords-engineering equipment; lash-up maintenance technology; fault diagnosis; damnification evaluation

\section{Introduction}

Engineering equipment lash-up maintenance, including activities of rapidly recovering equipment capabilities by applying lash-up diagnosis and recovery technologies, is the important part of technical support of engineering equipment.

Referring to the study hotspots of present equipment lash-up maintenance technology and considering the real situation of research on lash-up maintenance technology of engineering equipment, the paper put forwards the theory frame of lash-up maintenance technology of engineering equipment. See Figure 1.

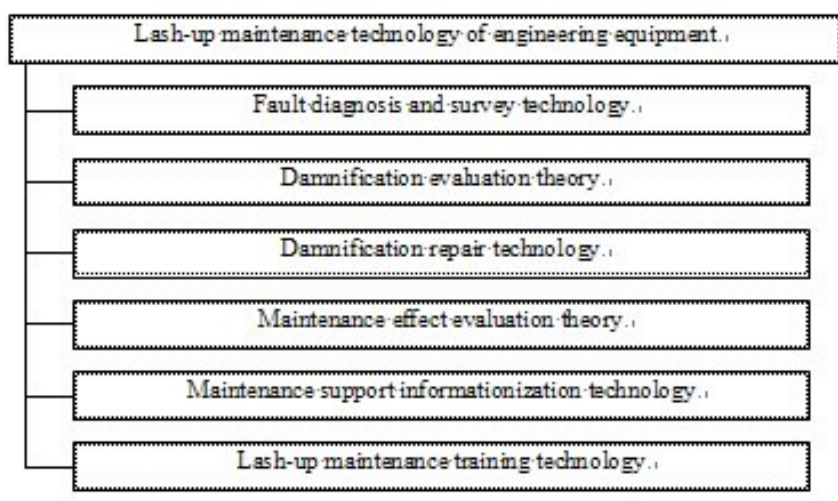

Fig. 1 The Theory Frame of Lash-up Maintenance Technology of Engineering Equipment

\section{Lash-up maintenance technology of engineering equipment}

\section{A Fault diagnosis and survey technology}

Aiming at modern engineering equipment more complicated system and more intensive technology, with "theater and rapid diagnosis" as its core, the research must be on virtual instrument technology, simulated diagnosis technology, key system situation inspection technology, comprehensive diagnosis technology of equipment fault based on information integration, fault prediction and healthy management technology. Accurate and reliable fault detection and insulation is the key of these technologies. Fault diagnosis and inspection system participates passively but not actively in the operation of equipment system.

\section{1) Fault diagnosis technology}

(1) Virtual instrument technology

Virtual instrument is a kind of instrument organically combined computer, connecting circuit and data analysis, processing communication and graphical user interface soft wares. Virtual instrument becoming mature and widely being applied to auto industry has opened broader prospects for its application to lash-up maintenance of engineering equipment.

\section{(2) Simulated diagnosis technology}

Simulated diagnosis technology is used for fault recurrence when the equipment is operating in same or similar working conditions, then fault can be found out and removed through check and analysis.

(3) Wavelet technology, neural network and grey system theory

Wavelet analysis technology, analyzing and processing signal time domain and frequency domain, has opened up a new method of picking up and quantificationally analyzing engineering equipment situation. Neutral network can establish the complicated mapping relations between fault symptom and fault model through its self-study function. 


\section{(4) Artificial intelligence technology}

Intelligent fault diagnosis system, based on expert system and artificial neutral network, is a system composed of human being, hard wares modern simulating brain function and necessary outer equipment, sensors and related soft wares supporting these hard wares. Artificial intelligence technology has been applied for fault diagnosis of hydraulic valve, pump and cylinder.

\section{(5) Remote fault diagnosis technology}

Remote fault diagnosis technology has its obvious features: first, it can analyze diagnosis tasks; second, it can obtain necessary information through remote control collecting, carry out remote fault diagnosis to engineering equipment in another place and transfer diagnosis result rapidly, third, it can manage several diagnosis tasks. These features are very important for lash-up maintenance of engineering equipment working in a big area and in bad working conditions.

\section{2) Fault survey technology}

Fault survey technology is applied through embedding or connecting sensors in equipment framework and spare parts to observe and reflect key parts' inner and outer environment, for example, the effect of pneumatic pressure, stress, thermal load and electromagnetic wave, spare part's leak or piercing.

\section{B Damnification evaluation theory}

Engineering equipment damnification evaluation and analysis is a method that based on equipment damnification data and combined characters of equipment lash-up maintenance, to analyze and judge fault spot rapidly and conclude the damnification evaluation. Engineering equipment damnification evaluation and analysis has two steps: first aiming at the final effect (namely damnification top affair) of engineering equipment damnification, following the structure of equipment damnification tree, determine the cause of damnification (namely middle affair) step by step by some inspection methods until find out the root cause (namely bottom affair) so as to form an engineering equipment damnification evaluation and analysis flow chart (or table), then in the light of damnification model and corresponding information in the table and integrating expert's experience and knowledge, decide whether the damnificated equipment could be repaired on the spot, needed manpower and time, technical situation and use limit after repair and necessary spare parts and equipment for repair, consequently result in engineering equipment damnification evaluation report (or conclusion). Damnification evaluation procedure is composed of damnification evaluation flow chart (or table) and damnification evaluation report (or conclusion).

Relevant evaluation methods includes expert system evaluation and failure tree evaluation.

\section{1) Expert system evaluation method}

Expert system is a kind of artificial intelligence computer program applying a great deal of human experts' knowledge and reasoning to figure out complicated real problems, which is widely used in artificial intelligence field. It differs from traditional technology which emphasizes the function of knowledge. It inputs human expert's specific knowledge into machine and make machine analyze and answer questions according to expert intention. Expert evaluation system mainly includes I/O interface, master computer, knowledge acquisition system, interpretative system, inference system and other modules. See Figure 2.

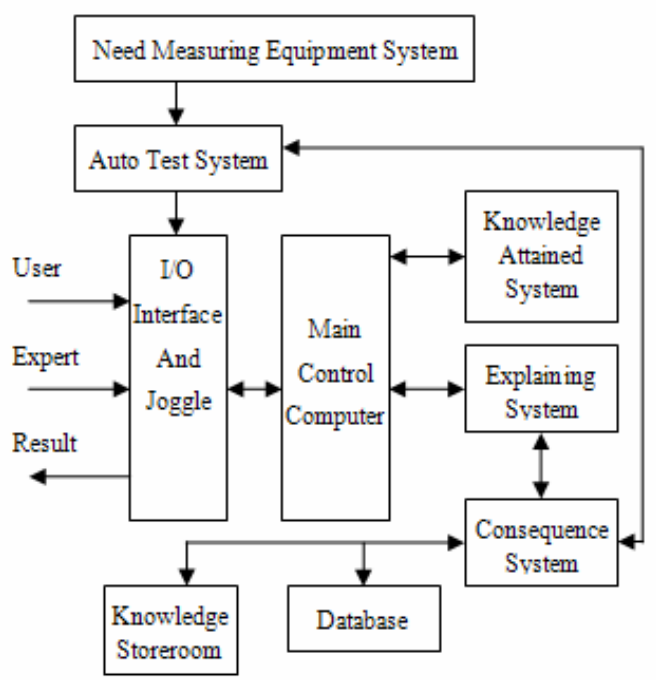

Fig. 2 Expert System Evaluation Flow Chart

\section{2) Failure tree analysis and evaluation method}

Failure tree analysis and evaluation method is a picture deduction analysis and evaluation method based on equipment inner damnification and is a kind of logic inference of failure event in certain condition. It is not restricted to common reliable analysis of the system but analyze in depth one or some certain failure situations so as to find out the logic relation between equipment component and system, judge damnification situation and degree. It includes the following steps: 1 . Determine equipment working principle, flow causal, damnification situation and logic relation. 2. Establish tree model, figure out the top affair accurately so as to infer the direct reason of damnification, thus a upside-down tree-shape logic causal chart come into being. 3. Provide result and application scope: mainly determine minimal cut set, namely the combination of component failure situations resulting in system damnification; determine importance, namely each component's responsibility for the system damnification; damnification probability of each component.

\section{Damnification repair technology}

Aiming at modern hi-tech engineering equipment and difficult lash-up maintenance, taking advanced maintenance technique as the core, mainly research the repair technology of equipment surface and new-type material structure, software-intensive equipment support technology, equipment erosion and fouling protection and handle technology, so as to realize the innovation of lash-up maintenance technology of engineering equipment and form advanced and systematical damnification repair technology.

Relevant technologies include: 1 . nano particle composite electro-brush plating technology, 2. high velocity arc-spray (HVAS), 3. nano solid lubrication dry film technology, 4. 
scratches quickly-filling technology, 5. nano anti-friction and in-situ dynamic self-repair technology, 6. ion compound infiltration technology, 7. Non-electric welding technology, 8. structure patch repair technology, 9. wear-resisting repair technology, 10. leak sealing technology, 11. Body-fitted sealing technology, 12. electronic equipment fast-cleaning technology, 13. Fast-cutting technology, 14. robot remanufacturing technology and so on.

\section{Maintenance effect evaluation theory}

Thinking of characters of engineering equipment lash-up maintenance, relatively suitable maintenance effect evaluation methods include comprehensive evaluation method, modeling and simulation method and so on.

\section{1) Comprehensive evaluation method}

Comprehensive evaluation method is a method combining qualitative and quantitative evaluation and used when evaluator do not understand some factors of lash-up maintenance, including analytic hierarchy process (AHP), fuzzy comprehensive evaluation, gray system amplitude analysis and DEA evaluation.

\section{2) Modeling and simulation method}

Lash-up maintenance effect evaluation based on modeling and simulation method can make use of simulation and modeling technology to establish systematical lash-up maintenance simulation model and carry on simulation experiment to obtain system data, then work out effect evaluation indexes through statistics and handling. Main methods include computer simulation method, system dynamics method, BCPNN method and other methods based on simulation tools.

\section{E Maintenance support informationization technology}

Aiming at the requirements of support accuracy, intensity and high efficiency of engineering equipment lash-up maintenance, the research must mainly be on crucial technology of equipment maintenance support information system, advanced communication and data transmission to develop informationization support and optimization technology based on network and computer.

\section{F Lash-up maintenance training technology}

Because of limited fund and manpower reduction, modular, simulating and simulation machine technology has become a development tendency of engineering equipment lash-up maintenance training technology. This improving technology must result in advanced lash-up maintenance training capabilities.

\section{1) Simulator technology}

Simulator technology can partly solve some problems in equipment lash-up maintenance training. Some engineering equipment component simulators can be established, even complicated full-function simulator can be established, which is operated like a real component, can be disassembled and also targeted.

\section{2) Virtual reality technology}

The established computer simulation environment through virtual reality technology contains interactive virtual objects, which enlarges human's cognitive range to analyze equipment's structure, working principle in virtual environment, even carry out lash-up maintenance directly. This can reduce training cost as well as have good training effect.

\section{3) Embedded simulation technology}

Embedded lash-up maintenance simulation of engineering equipment defines that in the precondition of not changing inner system structure of engineering equipment, embed the equipment having lash-up maintenance training simulation function into the whole engineering equipment system and make it as part of the system to carry on the training of engineering equipment and personnel.

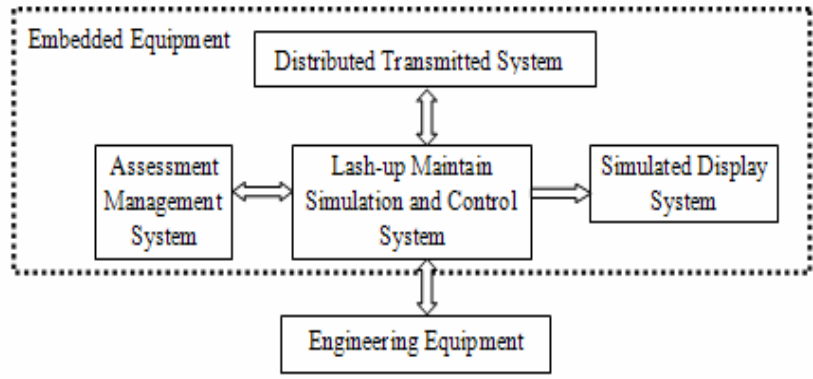

Fig. 3 Framework of Engineering Equipment Embedded Lash-up Maintenance Simulation System

\section{Conclusion}

In the future a large amount of technology-intensive and high-informationized engineering equipment will be put into use, which brings forward new requirements for engineering equipment technical support. This urgently requires to complete engineering equipment lash-up maintenance technical support system, and improve the research of lash-up maintenance technology and lash-up maintenance material to develop lash-up maintenance technology in the direction of being speedy, automating and intelligentized.

\section{References}

[1] X.L.Chen, M.Q.Xiao, X.Q.Wang: National Defence Technology, Vol.25 (2006) No.9, p.14-17.

[2] Z.W.Fu, P.Guo, T.Tan: O.I.Automation, Vol.27 (2008) No.1, p.41-42.

[3] S.K.Xin, X.J.Chen: Engineer Equipment Research, Vol.22 (2003) No.3, p.39-42.

[4] K.Feng, J.Zhong, J.Yan: Journal of PLA University of Science and Technology, Vol.5 (2004) No.2, p.72-74.

[5] Y.F.Shen, J.He: Engineering Equipment Operational Application(PLA Publicatoins, China 2007).

[6] A.Liu: Engineering Equipment (PLA Publicatoins, China 2002).

[7] Y.H.Wang: General Survey of Engineering Equipment Support (Military Science Publicatoins, China 2003). 